\title{
Viral Genome
}

National Cancer Institute

\section{Source}

National Cancer Institute. Viral Genome. NCI Thesaurus. Code C17253.

The total complement of hereditary material in a virus, which can be composed of either DNA or RNA. 